24 RESPECT (RECOMMENDED SUMMARY PLAN FOR EMERGENCY CARE AND TREATMENT) IN A PANDEMIC: THE IMPACT OF COVID-19 ON ADVANCE CARE PLANNING IN A UK UNIVERSITY HOSPITAL CARDIOLOGY DEPARTMENT

A Copley, M West, J Morris, F Hargreaves, MH Tayebjee. Leeds Teaching Hospitals NHS Trust, Department of Cardiology, Leeds General Infirmary

\subsection{6/spcare-2021-PCC.42}

Background Acute hospital clinicians play a key role in initiating discussions around advance care planning (ACP). Despite a widespread agreement that ACP is beneficial and facilitates patient-centred decision making, ACP prevalence in the acute hospital setting can be sporadic. COVID-19 created a heightened awareness of the need for early decision-making, in order to ensure patients received appropriate treatments, based on their general health, personal values and individual wishes.

Methods A retrospective study of patients admitted to a tertiary UK hospital Cardiology department during two discrete time periods; December 2019 and April 2020. Data was collected from electronic records and compared for significant differences between groups. This included the completion of ReSPECT forms, age, co-morbidities, frailty score and discharge diagnosis.

Results The study included 164 patients in total. There was no significant difference in age, co-morbidities or frailty between the groups. ReSPECT form completion significantly increased from December 2019 to April 2020 (9/84 (11\%) vs $39 / 80$ (49\%); $\mathrm{p}<0.0001)$. 25/26 (94\%) of COVID-19 positive patients had evidence of ReSPECT form discussion. For individuals with non-COVID-19 diagnoses, 14/54 (26\%) had evidence of ReSPECT form discussions.

Conclusions There was a statistically significant improvement in ACP during the first wave of COVID-19. Factors influencing this are likely to include heightened public and professional awareness of healthcare resources, an acknowledgement for rapid deterioration in patients with COVID-19, increased media coverage around the necessity for early decision-making, and a subsequent increase in patient expectation to discuss ACP. COVID-19 provides the opportunity to better establish meaningful ACP into acute hospital practice in the future. Further work is needed to assess the quality of the ACP process and individual patient experience. Increased familiarity with ACP, at both a public and professional level, could lead to enduring improvements in the facilitation of conversations and patient-centred decisions.

\section{TREATMENT ESCALATION PLANS IN CANCER CARE DURING THE COVID-19 PANDEMIC}

Oscar Short, Olivia Morley, Michael Davidson, Alexandra Hadjimichalis, Andrew Tweddle. The Royal Marsden NHS Foundation Trust

\subsection{6/spcare-2021-PCC.43}

Introduction Treatment escalation plans (TEP) enable the documentation of senior clinician-led and patient-centred advance care planning. The COVID-19 pandemic highlighted the importance of early decision-making regarding treatment escalation and early palliative care involvement. At the Royal
Marsden Hospital, a TEP form was created collaboratively by Palliative Care and Acute Oncology teams in response to the pandemic. It detailed current issues including cancer diagnosis, possible clinical interventions and prognosis.

Methods A retrospective study was performed of TEP completion in non-elective admissions from 6th-27th April 2020. We reviewed patient factors including prognosis, DNACPR status, palliative care involvement and patient outcomes using electronic patient records and TEP forms. A survey was emailed to all clinical staff for feedback on the TEP forms and their impact.

Results Of the 197 non-elective admissions, 105 (53.3\%) had a TEP completed. Compared to those without a TEP, patients who had a TEP completed were more likely to be on a non-curative than curative treatment pathway $(91 / 105$ $(86.6 \%)$ vs $50 / 92(54.3 \% ; \mathrm{p}<0.001 \ddot{\mathrm{I}}+2)$, have a documented DNACPR status $(78.1 \%$ vs $18.5 \%$; $<0.001$ İ $\$ 2)$, have palliative care input $(55.2 \%$ vs $25 \% ; \mathrm{p}<0.001 \quad \ddot{\mathrm{I}} \$ 2)$ or died ( $18.1 \%$ vs $6.5 \% ; p=0.015$ Ï $\$ 2)$ during admission. Suspected or confirmed COVID-19 infection did not impact upon TEP completion in this cohort. The online survey was completed by 59 staff members including 30 consultants. $74.5 \%$ respondents felt that the TEP form had a positive impact on patient care, with comments on possible refinements and improvements given.

Conclusions In a specialist cancer centre rates of completion of TEP forms were higher in non-curative patients receiving increased levels of palliative care input. The TEP form had a perceived positive impact on patient care amongst clinicians, although overall uptake was disappointing. We plan to update the TEP form in response to feedback, and re-audit after 6 months.

\section{THE END-OF-LIFE EXPERIENCES FOR CANCER PATIENTS WITH COVID-19; REFLECTIONS FROM 2 UK CANCER CENTRES}

Philippa McFarlane, Mary Miller, Matthew Carey, Angela Halley, Sophie Wilson, Nicola Wade, Joanne Droney. Royal Marsden NHS Foundation Trust (PM, AH, SW, NW, JD), The Churchill Hospital, Oxford University Hospital Trust, (MM, MC)

\subsection{6/spcare-2021-PCC.44}

Background The COVID-19 pandemic has seen a wealth of research examining the features of the disease. While large multicentre studies have detailed the implications of a cancer diagnosis and systemic anti-cancer therapy on mortality, little has been published regarding the end-of-life experiences for cancer patients dying COVID-19.

Aims To review the end-of-life care (EOLC) for patients with COVID-19 at 2 UK cancer centres.

Methods Prescriptions of anticipatory medications, opioids and continuous subcutaneous infusions of all non-ventilated inpatient deaths, $\mathrm{n}=28$.

Results The mean age was 67 (45-89), most patients were male and white British (18). 10 patients had recognised risk factors of cardiovascular disease and diabetes. 18 patients had metastatic disease and 23 were receiving palliative treatment. The most commonly recorded tumour type was GI (8). 12 patients were referred to palliative care (PC) for symptom control, while 10 were referred for EOLC. The mean number of PC reviews was 3.29 (range 0-10). Baseline 
IPOS scores on initial assessment at one centre $(\mathrm{N}=15)$, recorded the main symptoms as breathlessness, weakness and anxiety. Family anxiety was the highest scoring aspect of the IPOS. The medical management at the end-of-life was, however, generally uncomplicated; the total opioid (oral morphine equivalent) and benzodiazepine doses administered in last 24 hours before death were relatively low, median dose (range) $31.25 \mathrm{mg} \quad(5-160 \mathrm{mg})$ and $15 \mathrm{mg} \quad(0-30 \mathrm{mg})$ respectively.

Conclusions Should a further surge of COVID-19 cases occur, many cancer patients dying with COVID-19 could be appropriately managed by non-specialist physicians supported by comprehensive guidelines, ward-based teaching and specialist palliative care input for more complex cases. High levels of family anxiety could be improved by enhanced family support and communication about end-of-life wishes and priorities; healthcare services should have a particular focus on supporting the shadow pandemic of loss.

\section{EVALUATING THE ROLE OF THE ROYAL FREE LONDON NHS FOUNDATION TRUST PALLIATIVE CARE TEAMS DURING THE COVID-19 PANDEMIC}

Rory Carrigan, Sophie Cakebread, Louise Schofield, Nicola Henawi, Jo Wilson. Royal Free London NHS Foundation Trust

\subsection{6/spcare-2021-PCC.45}

Background The SARS-Cov-2 pandemic resulted in a rapid and unprecedented shift in the number of patients admitted to hospitals. In this trust palliative care provide a 9-5, 7 day/ week liaison service. We evaluated the role of the palliative care services during the peak of the pandemic.

Methods We conducted a retrospective analysis of the demand on palliative care team (PCT) at the two acute hospital sites; Royal Free Hospital (RFH) and Barnet Hospital (BH). Trust referral data was recorded for a 6-week period between 18/03 and 29/04, 2020. Patient outcomes were documented on a standardised Excel-database. Clinical notes were audited at random to ensure quality of data capture.

Results During the period studied there were 597 deaths between both sites, $393(66 \%)$ of which were documented as Covid-19 related. BH referred 178 patients to the PCT, of which 90\% were Covid-19 related. RFH referred 99 patients, of which 58\% were Covid-19 related. Clinical support provided to the wards caring for the patients was predominately in person at $\mathrm{BH}(97 \%)$ and via telephone at RFH (76\%), with an average time to death after referral of 1.9 days at $\mathrm{BH}$ and 2.8 days at RFH. Approximately 16\% of patients at both sites were discharged to other services for on-going care or end-of-life-care at home. The majority of pharmacological interventions were as-required medication $(>85 \%)$ with fewer patients than anticipated needing a syringe driver $(<60 \%)$. Clinical notes confirmed that most patients became symptomatic quickly, died rapidly after referral and communication with families via telephone was well documented.

Conclusions The two PCTs had different clinical experiences, this can be explained by the populations that each site serves, the structures within the teams and their physical location in relation to the wards. Excellent patient outcomes remained the same suggesting that both PCTs adapted well, with further shared learning planned.

\section{A REVIEW OF CARDIOPULMONARY RESUSCITATION (CPR) AND TREATMENT ESCALATION PLAN (TEP) DECISION-MAKING IN AN ACUTE LONDON TRUST DURING THE COVID-19 PANDEMIC - AN AUDIT-BASED STUDY}

Amelia Parker, Eleanor Manners, Lucy Gudge, Ben Hardy, Dijay Dave, Kathleen Bonnici, Ruth Caulkin. Chelsea and Westminster NHS Foundation Trust

\subsection{6/spcare-2021-PCC.46}

Introduction National guidance early in the COVID-19 pandemic encouraged frontline hospital staff to have discussions and make early decisions with patients regarding appropriateness of CPR and other medical treatments if they were to deteriorate.

An audit of CPR and TEP decisions was carried out in our trust at the pandemic peak and four weeks later compared to data from the previous year.

Methods Admission data was used to determine the peak of COVID-19 admissions. Electronic records of all adult inpatients (excluding maternity and Emergency Department) were reviewed and any completed CPR decisions and TEPs on the $2 / 4 / 20$ (peak) and 30/4/20 analysed using the same criteria as previous CPR and TEP audit.

Results and Discussion There was a 3 -fold increase in patients with a CPR decision at the peak (77\%) of the pandemic, compared with pre-pandemic (26\%), with a marked increase in number of decisions 'For CPR' from 1\% pre-pandemic to $33 \%$ at peak. However, this increase in number of CPR decisions reduced 4 -weeks post the peak to $58 \%$ as the pressure on admissions and ICU beds decreased.

The number of patients with a 'No CPR' decision with a TEP increased from $59 \%$ pre-pandemic to $88 \%$ at the peak suggesting an increased focus on reviewing benefits of treatments such as ventilation together with a CPR decision. This declined to $53 \%$, below pre-pandemic levels, at 4-weeks.

$70 \%$ of CPR decisions were made within 2 days of admission at the peak which declined to $65 \%$ 4-weeks later.

Conclusions At the peak of COVID-19 related admissions, the data set shows a marked increase in CPR decisions and completed TEPs compared to pre-pandemic baseline, indicating that the importance of such conversations and prioritisation of resources became a focus for healthcare professionals. The increase was not, however, sustained. Maintaining focus on early decision-making remains the challenge.

\section{DISCUSSIONS OF PREFERRED PLACE OF DEATH IN SECONDARY CARE DURING THE COVID-19 PANDEMIC}

Ruth Porther, Karishma Tailor, Prateek Choudhary, Julia McLaughlin, Shruti Lakra. Aneurin Bevan Health Board

\subsection{6/spcare-2021-PCC. 47}

Objectives Achieving Preferred Place of Death (PPD) may form part of a 'good death' for some patients and loved ones. In many circumstances, it may not be possible to facilitate a death outside of hospital during the COVID-19 pandemic but this does not exclude the need for constructive discussions of PPD in order to address this. The aim of this audit is to determine whether PPD is being discussed in a secondary care setting alongside Treatment Escalation Plans (TEPs) and explore influencing factors. 